\title{
HUMAN-LIKE MOTION FROM PHYSIOLOGICALLY-BASED POTENTIAL FIELD
}

\author{
O. Khatib, J. Warren, V. De Sapio, L. Sentis \\ Artificial Intelligence Laboratory, Department of Computer Science, Stanford University, \\ USA
}

The challenge of synthesizing motion behaviors is a long-standing problem in robotics. With the recent advent of complex humanoid systems, this challenge grows ever demanding. Due to their anthropomorphic design, humanoids should move in a human-like manner to facilitate movement within man-made environments and to accommodate interaction with their biological counterparts. Common control strategies for emulating human motion involve generating joint space trajectories or learning specific motions, but these approaches require off-line computations and do not generalize well to related tasks. There is a pressing need for a framework where natural motion is generated in real-time for a large range of tasks.

From observations of human motion, it is apparent that people perform many different behaviors while executing a task. These secondary criteria are the essence of natural human motion, affecting not whether but how the task is achieved. With this as motivation, we have developed a prioritized multiple task control framework that in real-time dynamically decouples each task. Equipped with this control framework, we propose a general methodology to discover the innate subtasks of human motion. These behaviors are initially identified by hypothesis or direct observation. By designing targeted motion capture experiments that excite these behaviors, we then confirm or refute their presence and ascertain their importance relative to other tasks.

We will illustrate this methodology by examining the muscular effort required to perform positioning tasks. In general terms, it is evident that 
humans avoid overexertion in commonplace motions. However, this concept is not easily quantified, nor is its relative importance to other behaviors clear. By utilizing biomechanical models for neuromuscular dynamics and control, we demonstrate that humans seek to minimize the muscular effort (normalized by torque capacity) needed to compensate for gravity while performing positioning tasks. While there are additional behaviors that characterize human motion, we believe that effort minimization is particularly significant. To this end, we conducted preliminary experiments to investigate the manner in which humans address muscular effort while performing tasks. In these experiments, we observe a strong trend that the gravity torques are balanced over the various joints in a manner that is correlated to the available torque capacity at each joint.

Based on these observations, and past use of muscle-level weighted norm criteria in the biomechanics community, we conjecture that postural motion involves the minimization of a muscle effort potential. Our results suggest a robust relationship between human motion and task level minimization of this muscle effort potential. By using this potential to define the posture field, we can utilize the posture space to minimize effort in real-time while performing any primary task. Moreover, the flexibility of our control framework will easily incorporate other behaviors upon identification. By establishing this basis, we expect to capture the characteristics that drive most of natural human motion. 